\title{
SCALING RATIOS AND TRIANGLES IN SIEGEL DISKS
}

\author{
Xavier Buff and Christian Henriksen
}

AbstraCt. Let $f(z)=e^{2 i \pi \theta} z+z^{2}$, where $\theta$ is a quadratic irrational. McMullen proved that the Siegel disk for $f$ is self-similar about the critical point. We give a lower bound for the ratio of self-similarity, and we show that if $\theta=(\sqrt{5}-1) / 2$ is the golden mean, then there exists a triangle contained in the Siegel disk, and with one vertex at the critical point. This answers a 15 years old conjecture.

\section{Introduction}

Definition 1. The polynomial $P_{\theta}$ is defined by

$$
P_{\theta}(z)=e^{2 i \pi \theta} z+z^{2},
$$

where $\theta$ has continued fraction expansion

$$
\theta=\left[a_{1}, a_{2}, a_{3}, \ldots\right]=\frac{1}{a_{1}+\frac{1}{a_{2}+\frac{1}{a_{3}+\ddots}} .} .
$$

In the following, for $x \in \mathbb{R} / \mathbb{Z},\{x\}$ denotes the unique real number representing $x$ in $[-1 / 2,1 / 2]$, and

$$
\frac{p_{n}}{q_{n}}=\left[a_{1}, \ldots, a_{n}\right]
$$

denote the rational approximation to $\theta$ obtained by truncating its continued fraction.

In 1942, Siegel [Si] proved that when $\theta$ is a diophantine number, the polynomial $P_{\theta}$ is conformally conjugate to a rotation near the origin. The maximal domain $D$ on which this conjugacy is defined is called the Siegel disk for $P_{\theta}$. It is the Fatou component of $P_{\theta}$ containing 0 . In particular, this result holds when $\theta$ is of bounded type, i.e., $\sup a_{i}<\infty$.

In 1986, Herman $[\mathrm{H}]$ and Świątek $[\mathrm{Sw}]$ proved that when $\theta$ is of bounded type, the boundary $\partial D$ of the Siegel disk is a quasi-circle containing the critical point $\omega_{\theta}=-e^{2 i \pi \theta} / 2$. The proof is based on a quasi-conformal surgery due to Ghys and Douady (see $[\mathrm{D}])$. In 1993, Petersen $[\mathrm{P}]$ proved that the Julia set $J\left(P_{\theta}\right)$ has Lebesgue measure zero, and is locally connected.

Received February 25, 1999.

Keywords: Holomorphic dynamics, Siegel disk, self-similarity. 
In 1997, McMullen [McM2] obtained results concerning the geometry of the Julia set $J\left(P_{\theta}\right)$. In particular, he proved that if $\theta$ is a quadratic irrational, then the boundary of the Siegel disk for $P_{\theta}$ is self-similar about the critical point. This result was conjectured and observed numerically more than a decade ago by Manton, Nauenberg and Widom [MN] [W].

The number $\theta$ is a quadratic irrational if and only if the continued fraction of $\theta$ is preperiodic. In that case, the rotation $x \mapsto x+\theta, x \in \mathbb{R} / \mathbb{Z}$ is self-similar (see [McM2] Theorem 2.1). More precisely, if $\theta=\left[a_{1}, a_{2}, \ldots\right]$, where $a_{n+s}=a_{n}$ for $n \geq N$, we can set

$$
\alpha=\theta_{N+1} \theta_{N+2} \ldots \theta_{N+s},
$$

where $\theta_{i}=\left[a_{i}, a_{i+1}, a_{i+2}, \ldots\right]$. Then for $n \geq N$,

$$
\left\{q_{n+s} \theta\right\}=(-1)^{s} \alpha \cdot\left\{q_{n} \theta\right\} .
$$

Our first goal is to prove the following result.

Theorem 1. Let $\theta=\left[a_{1}, a_{2}, \ldots\right]$, where $a_{n+s}=a_{n}$ for $n \geq N$, be a quadratic irrational and $\lambda \in \mathbb{D}^{*}$ be the scaling ratio for the self-similarity of the Siegel disk of $P_{\theta}$ about the critical point. Besides, let $\alpha$ be defined as above. Then

$$
0<\alpha<|\lambda|<1 \text {. }
$$

McMullen mentioned to us that this bound on $\lambda$ in terms of $\alpha$ via a modulus estimate is very similar to Bers' inequality for quasifuchsian groups; there one knows that the length of a hyperbolic geodesic in $Q(X, Y)$ is bounded by the hyperbolic length of the corresponding geodesic on $\mathrm{X}$ or $\mathrm{Y}$ (see [B] Theorem 3 and [McM3] Prop. 6.4).

In [McM2] (corollary 7.5), McMullen also shows that when the continued fraction expansion of $\theta$ has odd period, then the boundary of the Siegel disk does not spiral about the critical point. This means that any continuous branch of $\arg \left(z-\omega_{\theta}\right)$ defined along $\partial D \backslash\left\{\omega_{\theta}\right\}$ is bounded. In particular, this result holds for the golden mean Siegel disk, where $\theta=(\sqrt{5}-1) / 2=[1,1,1, \ldots]$. Our second result is the following.

Theorem 2. Using the same notations, if $-\pi / \log \left(\alpha^{2}\right)>1 / 2$, then the Siegel disk of the polynomial $P_{\theta}$, contains a triangle with one vertex at $\omega_{\theta}$.

Corollary 1. The Siegel disk of the polynomial $P_{\theta}, \theta=(\sqrt{5}-1) / 2$, contains a triangle with one vertex at $\omega_{\theta}$.

The corollary is immediate since for $\theta=(\sqrt{5}-1) / 2=[1,1,1, \ldots]$ we have $\alpha=\theta=(\sqrt{5}-1) / 2$, and

$$
-\frac{\pi}{\log \left(\alpha^{2}\right)} \sim 3.264251306>\frac{1}{2} .
$$

On Figure 1, we have drawn the filled-in Julia set of the polynomial $P_{\theta}, \theta=$ $(\sqrt{5}-1) / 2$. We have also zoomed near the critical point $\omega_{\theta}$ to show the selfsimilarity of the boundary of the Siegel disk. 

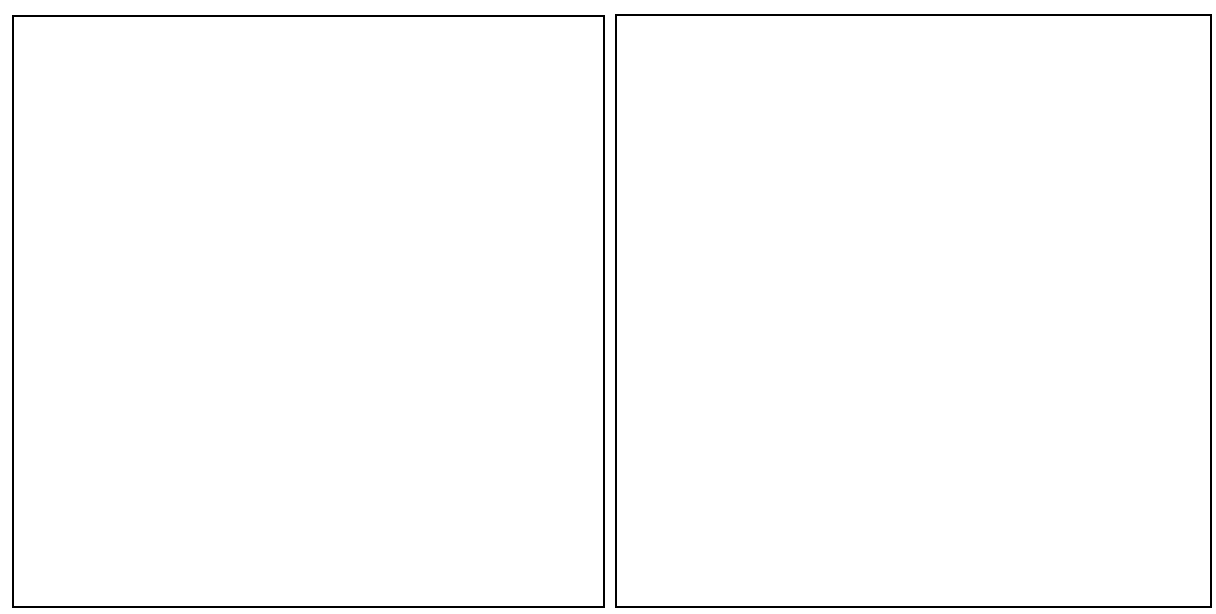

Figure 1. The filled-in Julia set of the polynomial $P_{\theta}$, $\theta=(\sqrt{5}-1) / 2$.

\section{The scaling ratio}

In the following, $\theta=\left[a_{1}, a_{2}, \ldots\right]$, where $a_{n+s}=a_{n}$ for $n \geq N$, will always be a quadratic irrational. We denote by $R_{\theta}(z)=e^{2 i \pi \theta} z$ the rotation of angle $\theta$. The polynomial $P_{\theta}$ has a Siegel disk $D$, and the conformal mapping $\phi: D \rightarrow \mathbb{D}$, linearizes $P_{\theta}$, i.e. conjugates $P_{\theta}$ to the rotation $R_{\theta}$. By results of Herman and Świątek, this conjugacy extends to a quasi-symmetric conjugacy $\phi: \partial D \rightarrow S^{1}$. Since $\phi$ is unique up to rotation, we can normalize it so that it maps $\omega_{\theta} \in \partial D$ to $1 \in S^{1}$ (see Figure 2).
$P_{\theta}$
$\omega_{\theta}$
$R_{\theta}$

$D$

$\mathbb{D} \quad 1$

$\phi$

FIgURE 2. The linearizing map $\phi: \bar{D} \rightarrow \overline{\mathbb{D}}$ sending $\omega_{\theta}$ to 1.

Now, recall that

- $p_{n} / q_{n}=\left[a_{1}, \ldots, a_{n}\right]$ is the rational approximation to $\theta$ obtained by truncating its continued fraction,

- $\theta_{i}=\left[a_{i}, a_{i+1}, a_{i+2}, \ldots\right]$, and

- $\alpha=\theta_{N+1} \theta_{N+2} \ldots \theta_{N+s}$. 
In [McM2], McMullen proves that for $n \geq N$,

$$
\left\{q_{n+s} \theta\right\}=(-1)^{s} \alpha\left\{q_{n} \theta\right\} .
$$

It follows that in a neighborhood of $z=1$, the contraction

$$
\begin{aligned}
& z \mapsto z^{\alpha}, \quad \text { for } s \text { even, and } \\
& z \mapsto \bar{z}^{\alpha}, \quad \text { for } s \text { odd, }
\end{aligned}
$$

conjugates $R_{\theta}^{q_{n}}$ to $R_{\theta}^{q_{n+s}}$, for $n \geq N$. Let us prove it for $s$ odd. For $z$ in a sufficiently small neighborhood of 1 , we have

$$
{\overline{R_{\theta}^{q_{n}}(z)}}^{\alpha}={\overline{e^{2 \pi i q_{n} \theta} \bar{z}}}^{\alpha}=\left(e^{-2 \pi i\left\{q_{n} \theta\right\}} \bar{z}^{\alpha}=e^{-2 \pi i \alpha\left\{q_{n} \theta\right\}} \bar{z}^{\alpha}=R_{\theta}^{q_{n+s}}\left(\bar{z}^{\alpha}\right) .\right.
$$

In [McM2] (Theorem 7.1), McMullen proves that there is a neighborhood $U$ of $\omega_{\theta}$ and a constant $\varepsilon>0$ such that for all $z \in U \cap \bar{D}$, the mapping $\psi$ defined by

$$
\psi(z)= \begin{cases}\phi^{-1}\left([\phi(z)]^{\alpha}\right), & \text { for } s \text { even, and } \\ \phi^{-1}\left([\overline{\phi(z)}]^{\alpha}\right), & \text { for } s \text { odd }\end{cases}
$$

- is well defined,

- satisfies the expansion

$$
\psi(z)= \begin{cases}\omega_{\theta}+\lambda\left(z-\omega_{\theta}\right)+\mathcal{O}\left(\left|z-\omega_{\theta}\right|^{1+\varepsilon}\right), & \text { for s even, or } \\ \omega_{\theta}+\lambda \overline{\left(z-\omega_{\theta}\right)}+\mathcal{O}\left(\left|z-\omega_{\theta}\right|^{1+\varepsilon}\right), & \text { for } s \text { odd },\end{cases}
$$

with $0<|\lambda|<1$, and,

- conjugates $P_{\theta}^{q_{n}}$ to $P_{\theta}^{q_{n+s}}$.

The main difficulty is to prove that $\psi$ is $C^{1+\varepsilon}$ at $\omega_{\theta}$.

Now let us define the scaling map

$$
\Lambda(z)= \begin{cases}\omega_{\theta}+\lambda\left(z-\omega_{\theta}\right), & \text { for s even, or } \\ \omega_{\theta}+\lambda \overline{\left(z-\omega_{\theta}\right)}, & \text { for } s \text { odd }\end{cases}
$$

For conveniency, we will use the spherical metric

$$
d \sigma(z)=\frac{|d z|}{1+\left|z-\omega_{\theta}\right|^{2}}
$$

(instead of the usual $|d z| /\left(1+|z|^{2}\right)$ ). Then the distance between two points $x$ and $y$ in $\mathbb{P}^{1}$ satisfy

$$
\sigma(x, y) \leq \inf \left(|x-y|, \frac{1}{\left|x-\omega_{\theta}\right|}+\frac{1}{\left|y-\omega_{\theta}\right|}\right) .
$$

This spherical metric enables us to define a Hausdorff distance $d_{H}$ between compact subsets of the sphere. McMullen shows the following theorem. 
Theorem 3. McMullen ([McM2], COROllary 7.3) The blow-ups $S_{n}=\Lambda^{-n}(\partial D)$ of the boundary of the Siegel disk converge to a $\Lambda$-invariant quasi-circle through $\infty$, for the Hausdorff topology on compact subsets of the sphere.

Proof. Indeed, there exists constants $C_{1}$ and $\delta>0$ such that for all $n$ large enough

$$
d_{H}\left(S_{n}, S_{n+1}\right)<C_{1}|\lambda|^{n \delta} .
$$

To see that, we need to prove that

- for any $x \in S_{n}$ there exists a $y$ in $S_{n+1}$ with $\sigma(x, y)<C_{1}|\lambda|^{n \delta}$, and

- for any $y$ in $S_{n+1}$ there exists a $x \in S_{n}$ with $\sigma(x, y)<C_{1}|\lambda|^{n \delta}$.

We will only prove the first point; a similar argument works for the second one. We first choose a constant $C$ and an open neighborhood $U^{\prime}$ of $\omega_{\theta}$ sufficiently small so that $\psi\left(U^{\prime}\right) \subset U^{\prime}$, and so that for any $z \in U^{\prime}$,

$$
|\psi(z)-\Lambda(z)|<C\left|z-\omega_{\theta}\right|^{1+\varepsilon} \text {. }
$$

We then set

$$
\delta=\frac{1}{2}\left(1-\frac{1}{1+\varepsilon}\right),
$$

which is positive. Observe that for all $n$ large enough, the ball $B_{n}$ centered at $\omega_{\theta}$ with radius $|\lambda|^{n(1-\delta)}$ is contained in $U^{\prime}$. Then, for any $x \in S_{n}, \Lambda^{n}(x)$ belongs to $\partial D$ and

- either $\Lambda^{n}(x) \in B_{n}, \psi\left(\Lambda^{n}(x)\right) \in \partial D$ and $y=\Lambda^{-(n+1)}\left(\psi\left(\Lambda^{n}(x)\right)\right.$ belongs to $S_{n+1}$; then a simple computation gives

$$
\sigma(x, y)<\frac{C}{|\lambda|}|\lambda|^{n \delta},
$$

- or $\Lambda^{n}(x) \notin B_{n}$ and $y=\Lambda^{-1}(x)$ belongs to $S_{n+1}$; moreover

$$
\sigma(x, y) \leq \frac{1}{\left|x-\omega_{\theta}\right|}+\frac{1}{\left|y-\omega_{\theta}\right|} \leq 2|\lambda|^{n \delta}
$$

Hence $d_{H}\left(S_{n}, S_{n+1}\right)$ is decreasing geometrically and the sequence $S_{n}$ is converging for the Hausdorff topology to a limit $\mathcal{S}$ which has to be $\Lambda$-invariant. Since the sets $S_{n}$ are all $K$ quasi-circles with the same $K$ (they are mapped onto each other by the scaling map $\Lambda$ ), the limit is also a $K$ quasi-circle.

Since $P_{\theta}$ is a quadratic polynomial, the Siegel disk $D$ has one preimage $D^{\prime} \neq D$ which is symmetric to $D$ with respect to $\omega_{\theta}$. The blow-ups $\Lambda^{-n}\left(D^{\prime}\right)$ and $\Lambda^{-n}(D)$ both converge, for the Hausdorff topology on compact subsets of the sphere, to $\Lambda$ invariant quasi-disks $\mathcal{D}$ (bounded by the quasi-circle $\mathcal{S}$ ) and $\mathcal{D}^{\prime}$ passing through $\infty$ and $\omega_{\theta}$ (see Figure 1). In particular, observe that $\mathcal{D} / \Lambda^{2}$ and $\mathcal{D}^{\prime} / \Lambda^{2}$ are two annuli in the torus $\left(\mathbb{C} \backslash\left\{\omega_{\theta}\right\}\right) / \Lambda^{2}$. We consider $\Lambda^{2}$ instead of $\Lambda$, because when $s$ is odd, $\Lambda$ is orientation reversing. Notice that when $s$ is even, this torus is conformally equivalent to $\mathbb{C}^{*} / \lambda^{2}$, and when $s$ is odd, this torus is conformally 
equivalent to the torus $\mathbb{C}^{*} /(\lambda \bar{\lambda})$. Besides, the annuli are conformally equivalent. Let

$$
M=\bmod \left(\mathcal{D} / \Lambda^{2}\right)=\bmod \left(\mathcal{D}^{\prime} / \Lambda^{2}\right)
$$

be their modulus.

The key-point in this paper is that we can compute the exact value of the modulus $M$.

Lemma 1. The modulus $M$ is equal to $-\pi / \log \left(\alpha^{2}\right)$.

Proof. Indeed, we can define the scaling map

$$
A(z)= \begin{cases}1+\alpha(z-1), & \text { for } s \text { even, and } \\ 1+\alpha \overline{(z-1)}, & \text { for } s \text { odd } .\end{cases}
$$

It is the differential at 1 of the contraction which conjugates $R_{\theta}^{q_{n}}$ to $R_{\theta}^{q_{n+s}}$, for $n \geq N$. Then

$$
\phi_{n}=A^{-n} \circ \phi \circ \Lambda^{n}
$$

is a conformal equivalence between $\Lambda^{-n}(D)$ and $A^{-n}(\mathbb{D})$, which extends quasisymmetrically to a map $\phi_{n}: \Lambda^{-n}(\partial D) \rightarrow A^{-n}(\partial \mathbb{D})$. Besides, $\phi_{n}\left(\omega_{\theta}\right)=1$, and

$$
A \circ \phi_{n+1}=\phi_{n} \circ \Lambda \text {. }
$$

By Caratheodory's convergence theorem, the sequence $\phi_{n}$ converges when $n$ tends to infinity, to a conformal map

$$
\phi_{\infty}: \mathcal{D} \rightarrow \mathbb{H}=\{z \in \mathbb{C} \mid \operatorname{Re}(z)<1\},
$$

such that $A \circ \phi_{\infty}=\phi_{\infty} \circ \Lambda$ (see [McM2] Theorem 8.1, statement 7). In particular, we see that the annulus $\mathcal{D} / \Lambda^{2}$ is isomorphic to the annulus $\mathbb{H} / A^{2}$. This last annulus has a modulus $M=-\pi / \log \left(\alpha^{2}\right)$.

We will now use a classical inequality on annuli embedded in a torus.

Lemma 2. Let $A_{i} \subset \mathbb{T}$, be disjoint annuli embedded in a torus

$$
\mathbb{T}=\mathbb{C} /(2 \pi i \mathbb{Z}+\tau \mathbb{Z}), \quad \operatorname{Re}(\tau)>0 .
$$

The segment $[0, \tau]$ projects to a simple closed curve $\gamma$ on $\mathbb{T}$. If the annuli $A_{i}$ are homotopic to $\gamma$, then

$$
\sum_{i=1}^{n} \bmod A_{i} \leq \frac{2 \pi R e(\tau)}{|\tau|^{2}}
$$

Proof. Let $B_{i}$ be the annulus

$$
\left\{z \mid 0<\operatorname{Im}(z)<h_{i}\right\} / \mathbb{Z}
$$

where $\mathbb{Z}$ acts by translations, with $h_{i}=\bmod B_{i}=\bmod A_{i}$, so that $A_{i}$ and $B_{i}$ are conformally equivalent. And let $f_{i}: B_{i} \rightarrow A_{i}$ be a conformal mapping. We can endow the torus $\mathbb{T}$ with the Euclidean metric; then the simple closed curve

$$
x \mapsto f_{i}(x, y), \quad 0 \leq x \leq 1
$$


has length at least $|\tau|$. Hence, we find

$$
\begin{aligned}
2 \pi \operatorname{Re}(\tau) & =\operatorname{Area}(\mathbb{T}) \\
& \geq \sum_{i} \operatorname{Area}\left(A_{i}\right) \\
& =\sum_{i} \int_{B_{i}}\left|f_{i}^{\prime}(x, y)\right|^{2} d x d y \\
& =\sum_{i} \int_{0}^{h_{i}}\left(\int_{0}^{1}\left|f_{i}^{\prime}(x, y)\right|^{2} d x\right) d y \\
& \geq \sum_{i} \int_{0}^{h_{i}}\left(\int_{0}^{1}\left|f_{i}^{\prime}(x, y)\right| d x\right)^{2} d y \\
& \geq \sum_{i} \int_{0}^{h_{i}}|\tau|^{2} d y \\
& =\sum_{i} h_{i}|\tau|^{2} .
\end{aligned}
$$

This is the required inequality.

In our case, $\tau$ is a branch of $\log \left(\lambda^{2}\right)$ when $s$ is even and of $\log (\lambda \bar{\lambda})$ when $n$ is odd. Using

$$
|\operatorname{Re}(\tau)|=-\log \left(|\lambda|^{2}\right)
$$

we get

$$
2 M \leq-\frac{2 \pi}{\log \left(|\lambda|^{2}\right)}
$$

Combining this with the exact value of the modulus $M$ gives

$$
-\frac{2 \pi}{\log \left(\alpha^{2}\right)} \leq-\frac{2 \pi}{\log \left(|\lambda|^{2}\right)}
$$

which is equivalent to

$$
\alpha<|\lambda|
$$

This proves Theorem 1 .

\section{Triangle in the golden Siegel disk}

We will now show that if $\theta=(\sqrt{5}-1) / 2$ is the golden mean, then the Siegel disk of $P_{\theta}$ contains a triangle with vertex at the critical point $\omega_{\theta}$. It is enough to show that the quasi-disk $\mathcal{D}$ contains a sector with vertex at $\omega_{\theta}$.

McMullen has already done the main step in that direction (see [McM2] corollary 7.5). He proved that when $s$ is odd (and in our case $s=1$ ), the boundary of the Siegel disk does not spiral about the critical point. That means that there exists a continuous branch of $\chi(z)=\log \left(z-\omega_{\theta}\right)$ defined on $\mathcal{D}$ with bounded imaginary part. Indeed, the condition that $\mathcal{D}$ is $\Lambda$-invariant implies that $\mathcal{D}$ is 
$\Lambda^{2}$-invariant. Now the scaling ratio of $\Lambda^{2}$ is $\lambda \bar{\lambda}$ and consequently the strip $\chi(\mathcal{D})$ is invariant by the translation $T(z)=z+\log (\lambda \bar{\lambda})$. This translation being real, the imaginary part of $\chi(z)$ is bounded when $z \in \mathcal{D}$.

To prove the existence of a sector in $\mathcal{D}$, it is enough to show that the strip $\chi(\mathcal{D})$ contains a horizontal band

$$
B=\left\{z \in \mathbb{C} \mid y_{1}<\operatorname{Im}(z)<y_{2}\right\}
$$

for some $y_{1}<y_{2}$ in $\mathbb{R}$ (see Figure 3 ).

On Figure 3, we have drawn the Julia set of the polynomial $P_{\theta}, \theta=(\sqrt{5}-1) / 2$ and its image under the map $\chi(z)=\log \left(z-\omega_{\theta}\right)$. It is very difficult to get a good picture of the Julia set $J\left(P_{\theta}\right)$ near the critical point $\omega_{\theta}$. However, it is possible to get a good idea of the boundary of the Siegel disk since the orbit of the critical point is dense in $\partial D$.



Figure 3. The filled-in Julia set of the polynomial $P_{\theta}$,

$\theta=(\sqrt{5}-1) / 2$ and its image under the map $\chi(z)=\log \left(z-\omega_{\theta}\right)$.

To prove that the strip $\chi(\mathcal{D})$ contains a horizontal band, recall that the quotient $\chi(\mathcal{D}) / T$ is an annulus of modulus $-\pi / \log \left(\alpha^{2}\right)$, where in our case $\alpha=\theta=(\sqrt{5}-1) / 2$. Since

$$
-\frac{\pi}{\log \left(\alpha^{2}\right)} \sim 3.264251306>\frac{1}{2},
$$

the existence of a sector in $\mathcal{D}$ is a consequence of the following lemma (see [McM1], Thm 2.1).

Lemma 3. Assume $S$ is a periodic strip of period $\tau \in \mathbb{R}$, i.e., $S+\tau=\tau$. If $S / \tau$ is an annulus of modulus

$$
\bmod (S / \tau)>\frac{1}{2},
$$


then $S$ contains a horizontal band

$$
B=\left\{z \in \mathbb{C} \mid y_{1}<\operatorname{Im}(z)<y_{2}\right\},
$$

for some $y_{1}<y_{2}$ in $\mathbb{R}$.

Proof. We will proceed by contradiction. If we cannot put a horizontal band in $S$, then there is a horizontal line which intersects both the upper boundary of $S$ and the lower boundary of $S$. We can assume without loss of generality that this line is the real axis. Under the mapping $z \mapsto e^{2 i \pi z / \tau}$, the strip projects to an annulus $A \in \mathbb{C}^{*}$, and the real axis projects to the unit circle $S^{1}=\{|z|=1\}$. Hence, the bounded component of $\mathbb{C}^{*} \backslash A$ contains a point $z_{1}$ of modulus 1 , and the unbounded component of $\mathbb{C}^{*} \backslash A$ contains a point $z_{2}$ of modulus 1 .

It is known (see $[\mathrm{LV}]$ page 56-65) that the modulus of an annulus separating the points 0 and $z_{1}$ from the points $z_{2}$ and $\infty$, with $\left|z_{1}\right|=\left|z_{2}\right|=1$ is bounded from above by the modulus of the annulus

$$
\left.\left.A_{\max }=\mathbb{C} \backslash(]-\infty,-1\right] \cup[0,1]\right) .
$$

In particular, when $\bmod (S / \tau)>\bmod \left(A_{\max }\right)$, we see that we get a contradiction. Douady indicated to us that this modulus is equal to $1 / 2$. Indeed, let us first consider a square pillow with side-length 1 . This pillow is isomorphic to $\mathbb{P}^{1}$. We can map two opposite corners to 0 and $\infty$, and a third corner to 1 . By symmetry of the square pillow, the remaining corner is mapped to -1 . The annulus $A_{\max }$ is then isomorphic to the pillow cut along two opposite sides. This is a cylinder with height 1 and circumference 2 . Hence the modulus of this cylinder is $1 / 2$.

Remark. We would like to mention that for the angle $\theta=[2,2,2,2, \ldots]=$ $\sqrt{2}-1$, the modulus of the corresponding annulus is

$$
-\pi / \log \left(\alpha^{2}\right) \sim 1.782213977>\frac{1}{2} .
$$

Hence, our proof enables us to conclude that there is still a triangle in the Siegel disk with vertex at the critical point (see Figure 4).

In fact we can conclude that there is an angle in the Siegel disk as long as $\theta=$ $[a, a, a, a, \ldots]$, with $a \leq 23$. Indeed, for $a=23$, we have $\alpha=\theta=(\sqrt{533}-23) / 2$, and

$$
-\pi / \log \left(\alpha^{2}\right) \sim .5006714845>\frac{1}{2} .
$$

On the other hand, when $a=24$, we get $\alpha=\theta=(\sqrt{580}-24) / 2$, and

$$
-\pi / \log \left(\alpha^{2}\right) \sim .4939944446<\frac{1}{2} .
$$

In this case, our proof does not enable us to conclude anything. We have drawn the corresponding Julia set on Figure 5. We have also drawn its image under the map $\chi(z)=\log \left(z-\omega_{\theta}\right)$ to show that the boundary of the Siegel disk "oscillates". It is a reason why our proof does not enable us to conclude anything, whereas 

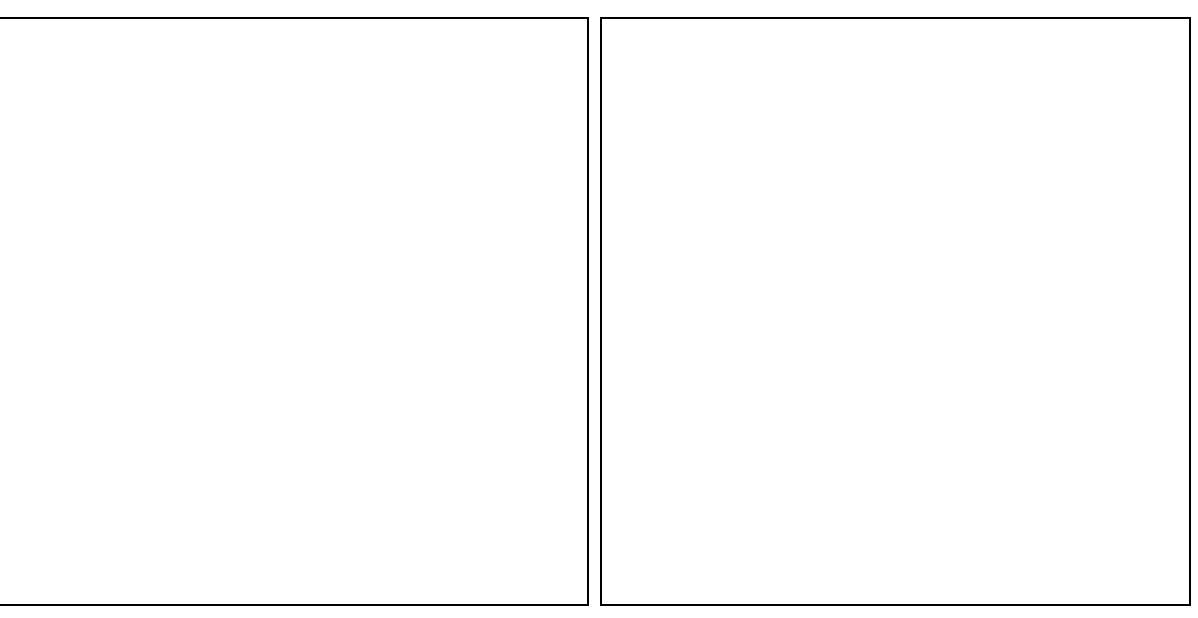

Figure 4. The filled-in Julia set of the polynomial $P_{\theta}$, $\theta=\sqrt{2}-1$.

it seems that one can put a triangle in the Siegel disk with vertex at the critical point.

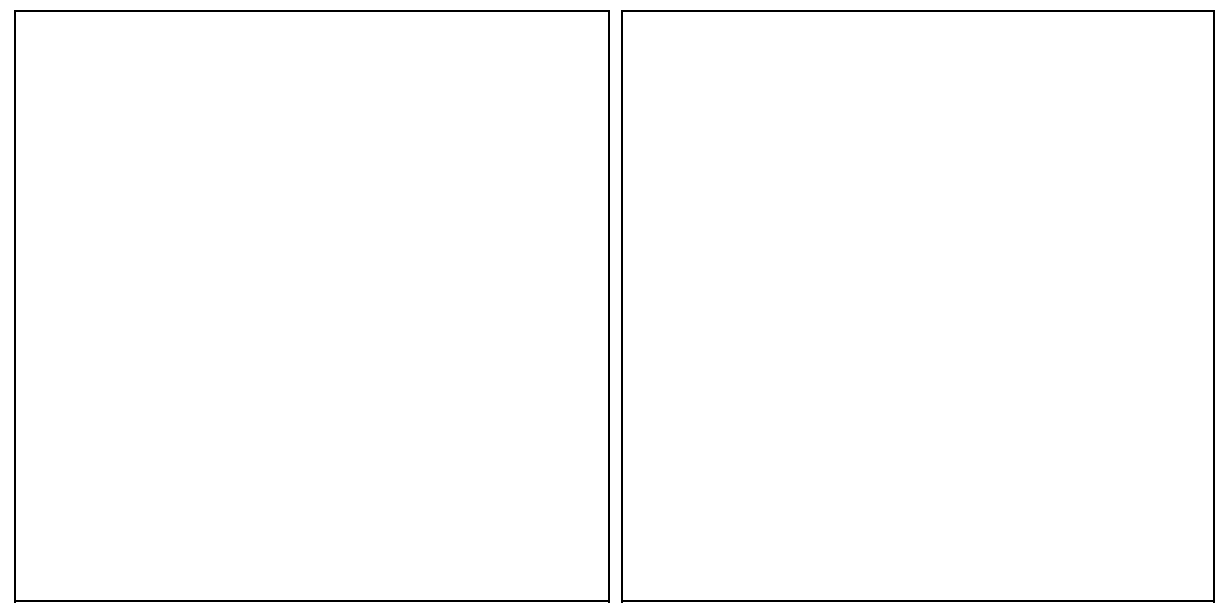

Figure 5. The filled-in Julia set of the polynomial $P_{\theta}$,

$\theta=[24,24,24, \ldots]$ and its image under the map $\chi(z)=\log \left(z-\omega_{\theta}\right)$.

\section{Questions}

We have seen that when the period of a quadratic irrational is odd, then the boundary of the corresponding Siegel disk does not spiral around the critical 
point.

Question 1. Is there a quadratic irrational $\theta$ such that the boundary of the Siegel disk of $P_{\theta}$ spirals?

Question 2. Does the boundary of the Siegel disk always spiral when the period of $\theta$ is even?

To answer those two questions, one has to show that the scaling ratio $\lambda \in \mathbb{D}^{*}$ is not a real number. Computer experiments suggests that for $\theta=$ $[2,1,2,1,2,1, \ldots]$, the ratio $\lambda$ is not real. Hence, the boundary of the Siegel disk spirals. We have drawn the Julia set of the polynomial $P_{\theta}$ for $\theta=$ $[2,1,2,1,2,1, \ldots]$, and its image under the map $\chi(z)=\log \left(z-\omega_{\theta}\right)$ (see Figure 6). It should be clear that the strip corresponding to the Siegel disk is not horizontal.

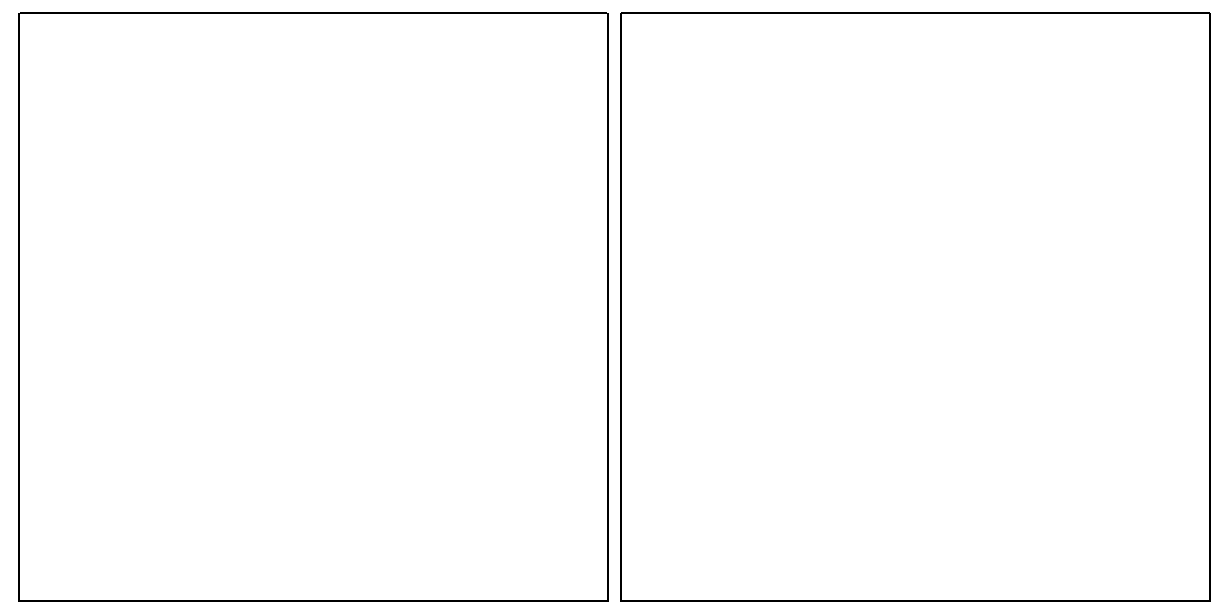

Figure 6 . The filled-in Julia set of the polynomial $P_{\theta}$, $\theta=[2,1,2,1,2,1 \ldots]$ and its image under the map $\chi(z)=\log \left(z-\omega_{\theta}\right)$.

Question 3. Is there a quadratic irrational $\theta$ with odd period, but for which there is no triangle with vertex at $\omega_{\theta}$ contained in the Siegel disk?

This problem seems to be related to question 1. Indeed, if there is a quadratic irrational such that the boundary of the Siegel disk of $P_{\theta}$ spirals, then the period $s$ of $\theta$ is even. Let us write $\theta=\left[a_{1}, \ldots, a_{s}, a_{1}, \ldots, a_{s}, \ldots\right]$. Now consider the quadratic irrational $\theta^{\prime}=\left[a_{1}^{\prime}, \ldots, a_{k s+1}^{\prime}, \ldots\right]$ of period $k s+1$, where $k$ is a large integer, and where

$$
\begin{aligned}
a_{1}^{\prime} & =a_{1}, \quad \text { and } \\
a_{i}^{\prime} & =a_{i-1}, \quad \text { if } \quad 2 \leq i \leq k s+1 .
\end{aligned}
$$


Then, one can expect that the boundary of the Siegel disk of $P_{\theta^{\prime}}$ will oscillate. On Figure 7, we have drawn the filled-in Julia set of the polynomial $P_{\theta}, \theta=$ $[50,50,1,50,50,1,50,50,1, \ldots]$ and its image under the map $\chi(z)=\log \left(z-\omega_{\theta}\right)$. It is very difficult to obtain a good picture of the boundary of the Siegel disk near the critical point. The dark region on Figure 7 corresponds to something we extrapolated.

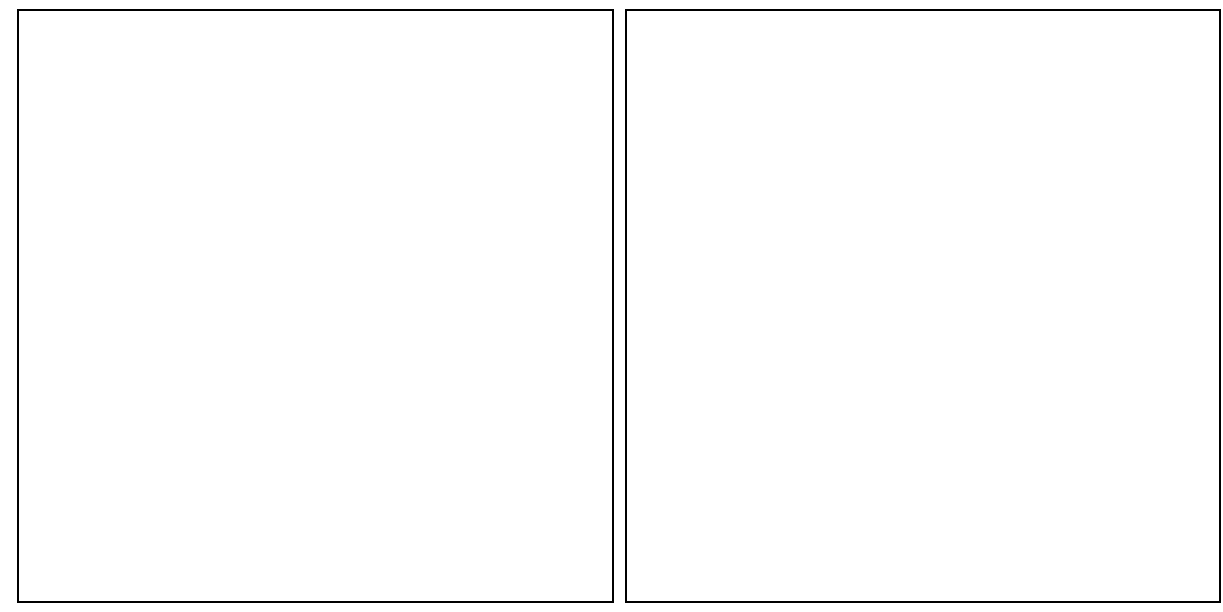

Figure 7 . The filled-in Julia set of the polynomial $P_{\theta}$, $\theta=[50,50,1,50,50,1,50,50,1, \ldots]$ and its image under the map $\chi(z)=\log \left(z-\omega_{\theta}\right)$.

Finally, we know that $|\lambda|<1$ for every quadratic irrational $\theta$. When we show that there is a triangle in the Siegel disk, we don't get any lower bound on the angle of the vertex which is at the critical point. We could get one if we knew that $|\lambda|$ is not too close to 1 .

Question 4. Is there a constant $\delta<1$ such that $|\lambda|<\delta$ for any quadratic irrational $\theta$ ?

We can even be more optimistic.

Question 5. Is there a constant $\delta<1$ such that $|\lambda|<\delta^{s}$, where $s$ is the period of the quadratic irrational $\theta$ ?

Finally, we would like to ask a last question, which seems to be a analog of Świątek's a-priori bounds for Blaschke fractions having an irrational rotation number:

Question 6. Are there constants $\delta_{1}<\delta_{2}<1$ such that $\left(\delta_{1}\right)^{s}<|\lambda|<\left(\delta_{2}\right)^{s}$, where $s$ is the period of the quadratic irrational $\theta$ ? 


\section{Acknowledgements}

We wish to thank Adrien Douady for mentioning this problem. We are grateful to Curt T. McMullen for carefully reading a first version of this paper and suggesting several improvements. We also want to thank John H. Hubbard, Misha Lyubich and Carsten L. Petersen for valuable comments and the Departments of Mathematics at Cornell University, at the Technical University of Denmark and at Université Paul Sabatier in Toulouse for hospitality during the research that went into this paper.

\section{References}

[B] L. Bers, On Boundaries of Teichmüller spaces and on Kleinian groups, Annals of Math. (2) 91 (1970), 570-600.

[D] A. Douady, Disques de Siegel et anneaux de Herman, Séminaire Bourbaki, Astérisque 152-153 (1986/87), 151-172.

[H] M. Herman, Conjugaison quasi-symétrique des difféomorphismes du cercle et applications aux disques singuliers de Siegel, Manuscript, 1986.

[LV] O. Lehto and K.I. Virtanen, Quasi-conformal mappings in the plane, Springer-Verlag, Berlin, 1973.

$[\mathrm{MN}]$ N.S. Manton and M. Nauenberg, Universal scaling behavior for iterated maps in the complex plane, Comm. Math. Phys. 89 (1983), 555-570.

[McM1] C.T. McMullen, Complex dynamics and renormalization, Annals of Mathematics Studies, 135., Princeton University Press, Princeton, NJ, 1994.

[McM2] _ Self-similarity of Siegel disks and Hausdorff dimension of Julia sets, Acta Math. 180 (1998), 247-292.

[McM3] _ Iteration on Teichmüller space, Inv. Math. 99 (1989), 425-454.

[P] C.L. Petersen, Local connectivity of some Julia sets containing a circle with an irrational rotation, Acta Math. 177 (1996), 163-224.

[Si] C.L. Siegel, Iteration of analytic functions, Ann. of math. (2) 43 (1942), 607-612.

[Sw] G. Świątek, Rational rotation numbers for maps of the circle, Comm. Math. Phys. 119 (1988), 109-128.

[W] M. Widom, Renormalization group analysis of quasi-periodicity in analytic maps, Comm. Math. Phys. 92 (1983), 121-136.

Université Paul Sabatier, Laboratoire Emile Picard, 31062 Toulouse Cedex, FRANCE

E-mail address: buff@picard.ups-tlse.fr

The Technical University of Denmark, Department of Mathematics, 2800 Lyngby, DENMARK

E-mail address: chris@mat.dtu.dk 